\title{
Biallelic variants in AGTPBP1, involved in tubulin deglutamylation, are associated with cerebellar degeneration and motor neuropathy
}

\author{
Ruth Sheffer $\mathbb{D}^{1} \cdot$ Michal Gur $^{1} \cdot$ Rebecca Brooks $^{2} \cdot$ Somaya Salah $^{1} \cdot$ Muhannad Daana $^{3} \cdot$ Nitay Fraenkel $^{4}$. \\ Eli Eisenstein ${ }^{5} \cdot$ Malcolm Rabie $^{6} \cdot$ Yoram Nevo $^{6} \cdot$ Chaim Jalas $^{7} \cdot$ Orly Elpeleg $^{1,8} \cdot$ Shimon Edvardson $^{8,9} \cdot$ Tamar Harel $^{1}$
}

Received: 26 October 2018 / Revised: 16 March 2019 / Accepted: 26 March 2019 / Published online: 11 April 2019

(c) European Society of Human Genetics 2019

\begin{abstract}
The ATP/GTP-Binding Protein $1(A G T P B P 1)$ gene $(\mathrm{OMIM} * 606830)$ catalyzes deglutamylation of polyglutamylated proteins, and its deficiency manifests by cerebellar ataxia and peripheral neuropathy in mice and lower motor neuron-like disease in sheep. In the mutant mice, cerebellar atrophy due to Purkinje cell degeneration is observed, likely due to increased tubulin polyglutamylation in affected brain areas. We report two unrelated individuals who presented with early onset cerebellar atrophy, developmental arrest with progressive muscle weakness, and feeding and respiratory difficulties, accompanied by severe motor neuronopathy. Whole exome sequencing followed by segregation analysis in the families and cDNA studies revealed deleterious biallelic variants in the AGTPBPI gene. We conclude that complete loss-of-function of AGTPBP1 in humans, just like in mice and sheep, is associated with cerebellar and motor neuron disease, reminiscent of Pontocerebellar Hypoplasia Type 1 ( $\mathrm{PCH} 1)$.
\end{abstract}

Supplementary information The online version of this article (https:// doi.org/10.1038/s41431-019-0400-y) contains supplementary material, which is available to authorized users.

\section{Ruth Sheffer}

Ruthsh@ hadassah.org.il

1 Department of Genetic and Metabolic Diseases, Hadassah-Hebrew University Medical Center, 9112001 Jerusalem, Israel

2 Pediatric Intensive Unit, Department of Pediatrics, Mount Scopus Hadassah-Hebrew University Medical Center, Jerusalem, Israel

3 Child Development Centers, Clalit and Maccabi Health Care Services, Jerusalem, Israel

4 Department of Respiratory Rehabilitation, Alyn Hospital, Jerusalem, Israel

5 Department of Pediatrics, Hadassah-Hebrew University Medical Center, Jerusalem, Israel

6 Institute of Child Neurology, Schneider Children's Medical Center of Israel, Tel Aviv University, Tel Aviv, Israel

7 Bonei Olam, Center for Rare Jewish Genetic Diseases, Brooklyn, NY, USA

8 Monique and Jacques Roboh Department of Genetic Research, Hadassah-Hebrew University Medical Center, Jerusalem, Israel

9 Pediatric Neurology Unit, Hadassah-Hebrew University Medical Center, Jerusalem, Israel

\section{Introduction}

Pontocerebellar hypoplasia $(\mathrm{PCH})$ is a group of rare, inherited progressive neurodegenerative disorders [1]. To date, 11 different subtypes have been defined by clinical, neuroradiological and genetic features. Common characteristics include hypoplasia/atrophy of the cerebellum and pons, progressive microcephaly, variable cerebral involvement and autosomal recessive inheritance. Affected individuals suffer from severe cognitive and motor deficits. Seventeen causative genes have been reported, most of which function in RNA processing or translation. The first patient with pontocerebellar hypoplasia $(\mathrm{PCH})$ and spinal motor neurodegeneration as seen in Werdnig-Hoffman disease (or spinal muscular atrophy, SMA) was reported in 1961 by Norman [2], followed by additional reports of similar patients [3, 4]. These reports enabled delineation of a distinct subtype of PCH characterized by pontocerebellar hypoplasia or atrophy with bulbar and spinal motor neurodegeneration, to be classified as PCH type 1. Heretofore, variants in five genes have been associated with PCH type 1, with $~ 50 \%$ of patients carrying variants in EXOSC3 [57]. EXOSC 3 encodes for component 3 of the exosome complex, which is involved in RNA processing, and variants are associated with variable degrees of cerebellar hypoplasia and pons involvement. 
Variants in genes encoding other components of the exosome, EXOSC8 and EXOSC9, are similarly associated with psychomotor retardation, spasticity, hearing and vision impairment and severe muscle weakness due to motor neuron degeneration [8, 9]. Brain imaging shows progressive cerebellar atrophy with relative sparing of the brainstem. Variants in genes encoding non-exosomal components were also associated with $\mathrm{PCH}$ and SMA-like disease, as in the vaccinia-related kinase 1 (VRK1) [10-12] and the SLC25A46 genes [13, 14]. VRK1 was previously reported to be of importance for the maintenance of amyloid- $\beta$ precursor protein [15], whereas SLC25A46 encodes a mitochondrial outer membrane carrier which is involved in mitochondrial fission and fusion and maintenance of the mitochondrial cristae [16]. Thus PCH1 is a common phenotype to perturbations in several essential, but seemingly unrelated, mechanisms.

Here we report on the results of molecular investigation of two affected individuals from two unrelated families, who suffered from cerebellar hypoplasia and SMA-like disease. Our data adds a new mechanism to this group of disorders.

\section{Materials and methods}

\section{Exome analysis}

Following informed consent, exome analysis was pursued on DNA extracted from whole blood. Exome analysis of patient 1 and his parents and of patient 2 was performed on exon targets captured using SureSelect Human All Exon 50 Mb V5 Kit (Agilent Technologies). Sequences were determined by HiSeq2500 (Illumina, San Diego, California, USA) as 100-125-bp paired-end runs, with a mean coverage of $75 \times$. The full sequencing methodology and variant interpretation protocol were previously described [17].

\section{Segregation analysis}

Amplicons containing the AGTPBPI variants were amplified by conventional PCR, and analyzed by Sanger dideoxy nucleotide sequencing. The following primers were used for Family 1: forward primer: 5'- TGCTCATCTGTTTAAA TGCAATG-3' and reverse primer: 5'-TTTTCCACAGTT AAGATGCCTG-3'. For Family 2, AGTPBP1 exon 16 (NM_001286715.1) was amplified with forward primer: $5^{\prime}$ -

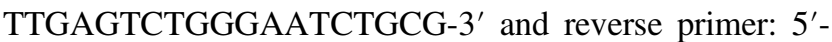
TTTTCCACAGTTAAGATGCCTG-3' . AGTPBPl exon 20 was amplified with forward primer: 5'-GACATTTCT AAAGCCCCAAAG-3' and reverse primer: 5'-AACCTG GACATTGTTATTTCAAC-3'. Exons were numbered sequentially according to their delineation in
NM_001286715.1, and as per UCSC genome browser (URL: genome.ucsc.edu) for this RefSeq transcript.

\section{RNA studies}

RNA was isolated from fresh lymphocytes of the patients, parents and control using Quick-RNA ${ }^{\mathrm{TM}}$ Whole Blood kit (Zymo Research) and cDNA was prepared using Maxima ${ }^{\mathrm{TM}}$ 1 st strand cDNA synthesis kit (Thermo Scientific). For Family 1, the region encompassing exons 14-16 of AGTPBP1 was amplified by PCR reaction using KAPA2G Fast HotStart Kit (KAPA Biosystems) with forward primer: 5'-TCAGAGGTGGCTTATCCCGA-3' and reverse primer: 5'-GAATCCACCATGGTCTGGCA-3'. The resultant fragments were separated by $2 \%(\mathrm{w} / \mathrm{v})$ agarose gel electrophoresis and their sequence determined by Sanger sequencing.

\section{Cloning for separation of alleles}

RNA and cDNA were prepared as above. The following primers were used to amplify an amplicon spanning both variants and to integrate an EcoRI restriction site: forward primer: 5'-taagcagaattcCACCATTCCAGAAGAGGGAG A-3' and reverse primer: 5'-taagcagaattcGACTTGGACT TTGCCACTGC-3'. The amplicon and pCS2+ vector were cut using EcoRI restriction enzyme and were ligated using T4 ligase. Clones were amplified by PCR and Sanger sequenced using the following primers: forward primer: $5^{\prime}$ CACCATTCCAGAAGAGGGAGA-3' and reverse primer: 5'-GACTTGGACTTTGCCACTGC-3'.

\section{Results}

\section{Clinical reports}

Clinical data is summarized in Table 1.

Family 1 (Fig. 1a, individual III-1)

The proband is a female firstborn child to healthy Palestinian Muslim Arab parents who are first cousins. She was born after 41 weeks of gestation by spontaneous vaginal delivery. Birthweight was $3280 \mathrm{~g}$, head circumference was $35 \mathrm{~cm}$ (50th percentile for age) and length was $50 \mathrm{~cm}$ (50th percentile for age). After birth she was noted to be hypotonic and have a weak cry, necessitating temporary respiratory assistance. Apgar score was 7 at $1 \mathrm{~min}$ and 9 at $5 \mathrm{~min}$. At the age of 2 months she continued to have weak sucking, axial hypotonia, poor head control and hyporeflexia, and was referred for metabolic and genetic assessment. Thyroid function tests, creatine kinase, ammonia, and 
Table 1 Clinical features of affected individuals

\begin{tabular}{|c|c|c|}
\hline Clinical feature & Family 1, Individual III-1 & Family 2, Individual II-6 \\
\hline Age at last evaluation & 15 months & 4 years 2 months \\
\hline Gender & $\mathrm{F}$ & M \\
\hline Ethnicity & $\begin{array}{l}\text { Palestinian, first cousin } \\
\text { parents }\end{array}$ & Ashkenazi Jewish \\
\hline Gestational week & 41 & 39 \\
\hline Birthweight & $3280 \mathrm{~g}(\mathrm{AGA})$ & $3500 \mathrm{~g}(\mathrm{AGA})$ \\
\hline Apgar scores (1/5 min) & $7 / 9$ & NA \\
\hline Respiratory distress & $+($ tracheostomy $)$ & + \\
\hline Mechanical ventilation & $\begin{array}{l}+ \text { (continuous since } \\
6 \text { months) }\end{array}$ & $\begin{array}{l}\text { Non-invasive mechanical ventilation at } \\
\text { night (since } 3 \text { years } 8 \text { months); oxygen } \\
\text { supplementation during the day }\end{array}$ \\
\hline $\begin{array}{l}\text { Mechanical insufflation- } \\
\text { exsufflation device }\end{array}$ & + & + \\
\hline Feeding difficulties & + (gastrostomy) & + (gastrostomy) \\
\hline $\begin{array}{l}\text { Recurrent aspirations; } \\
\text { recurrent pneumonia }\end{array}$ & + & + \\
\hline Poor head control & + & + \\
\hline Axial hypotonia & + & + \\
\hline Hyporeflexia & + & + \\
\hline Hearing & Normal & Normal \\
\hline Ophthalmological exam & Normal & Normal \\
\hline Lactate & Normal & NA \\
\hline Creatine kinase & Normal & Normal \\
\hline Progressive microcephaly & + & + \\
\hline MRI & $\begin{array}{l}\text { Enlarged CSF spaces, small } \\
\text { cerebellar hemispheres and } \\
\text { vermis }\end{array}$ & Small cerebellum \\
\hline Electrophysiology & $\begin{array}{l}\text { Severe motor neuropathy } \\
\text { (anterior horn cell disease) }\end{array}$ & NA \\
\hline Other & - & Hand tremor, tongue fasciculations \\
\hline$A G T P B P 1$ variants & c. $2342+2 \mathrm{~T}>\mathrm{G}$ [hom] & $\begin{array}{l}\text { c. } 2351 \mathrm{~A}>\mathrm{G} ; \text { p.Tyr784Cys [het]; } \\
\text { c.2998C >T; p.Arg1000Ter [het] }\end{array}$ \\
\hline
\end{tabular}

$\overline{A B R}$ auditory brain response, $A G A$ appropriate for gestational age, $C M A$ chromosomal microarray, het heterozygous, hom homozygous, $N A$ not available lactate levels were within normal limits. Auditory brain response was normal as was an ophthalmological examination. Brain MRI at 5 months of age showed enlarged CSF spaces, especially in the posterior fossa, with a relatively small volume of cerebellar hemispheres and vermis (Fig. 2a). Spinal muscular atrophy (SMA) due to deletion of the SMN1 gene was ruled out. Chromosomal microarray (CMA) revealed a normal female molecular karyotype with multiple homozygous regions. The child was hospitalized at age 4 months due to feeding difficulty, choking and failure to thrive and underwent insertion of a feeding gastrostomy. Respiratory distress necessitated intermittent assisted ventilation due to recurrent atelectasis. At 6 months of age, the child underwent insertion of a tracheostomy. Despite her neuromuscular difficulties, the child was alert and communicative. A progressive decrease in head circumference was noted (Fig. S1), with most recent head circumference measuring $43.2 \mathrm{~cm}$ at age 15 months ( $-2 \mathrm{SD})$.

Electrophysiological examination at 8 months of age revealed severe motor neuronopathy in both lower limbs and left arm (absent CMAPS, no motor responses with high stimulation). Sensory studies showed normal sensory nerve action potentials (SNAPS) for age (Table S1). Needle electromyography (EMG) showed widespread prominent neurogenic findings: active denervation $3-4 / 4+$ fibs \& psw's in both tibialis anterior and left posterior deltoid muscles, with complex repetitive discharges (CRDs) and myotonic discharges in some muscles, and reduced motor unit potential (MUP) recruitment in right tibialis anterior, right iliopsoas and left posterior deltoid muscles, giant MUPs $(10 \mathrm{mV})$ in right iliopsoas, and no volition in the left tibialis anterior muscle. Repetitive stimulation was unobtainable due to absent responses in all motor nerves. In 
A

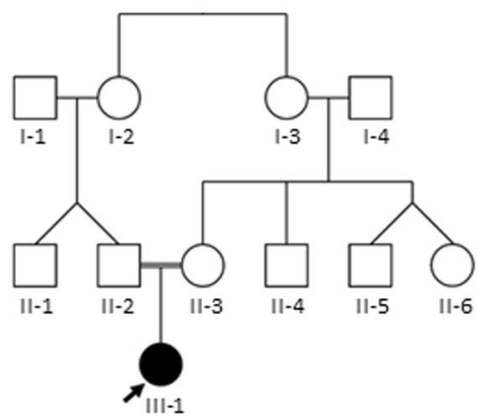

B

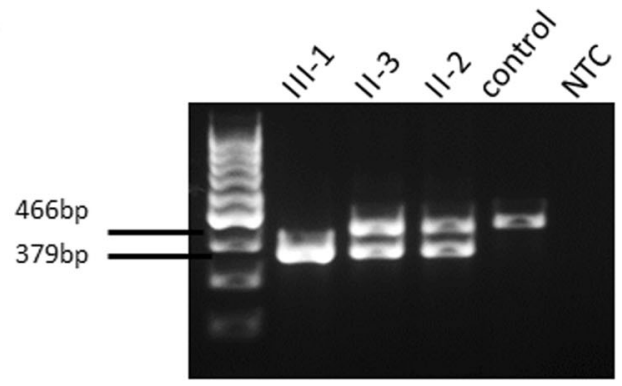

C

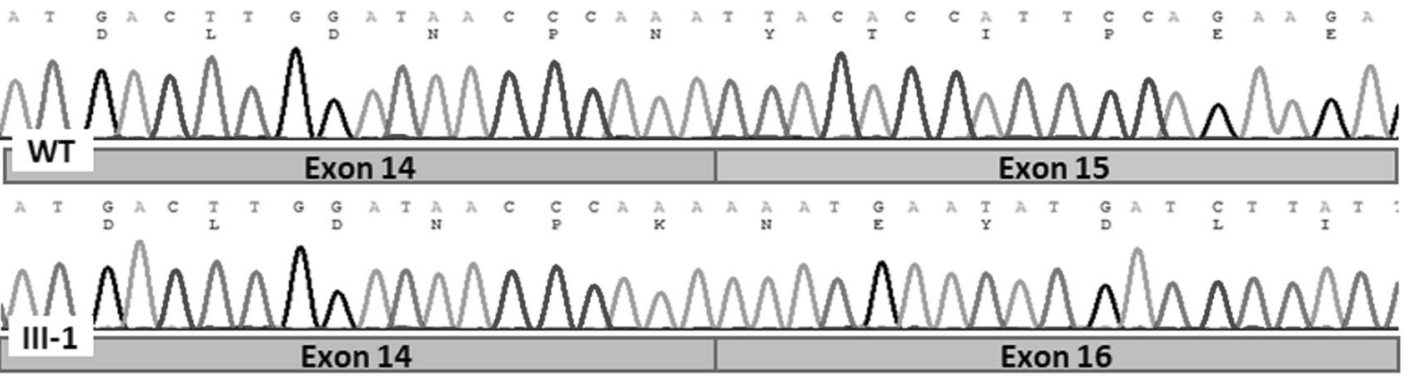

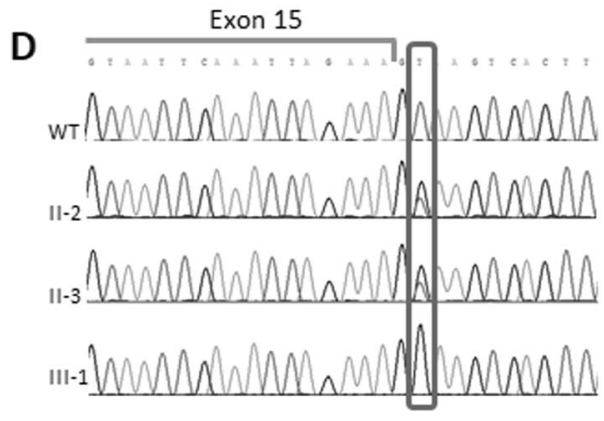

Fig. 1 Pedigree and molecular analysis of Family 1. a Pedigree indicating the affected child of first cousin parents. b PCR product amplified from cDNA of the proband (III-1), her parents (II-2 and II-3) and a healthy control. Last lane is the no-template control (NTC). c Sanger sequencing of cDNA from healthy control (upper panel) and individual III-1 (lower panel) showing skipping of exon 15 as a result

conclusion, the electrophysiological studies indicated a severe motor neuronopathy with widespread severe active denervation with late MUP recruitment and late chronic reinnervation, and normal sensory studies. These findings were compatible with anterior horn cell disease.

\section{Family 2 (Fig. 3a, individual II-6)}

The proband is a 50-months-old-male, the youngest child of 6, born to healthy unrelated Ashkenazi Jewish parents after 39 weeks of gestation, at a birth weight of $3500 \mathrm{~g}$. The perinatal course was uneventful. At 4 months of age, increasing difficulty in raising his head was noted and he began physiotherapy. At 6 months of age he could track faces and had a social smile though head control was poor and he could not roll over. Tremor of the hands was seen of the $c .2342+2 \mathrm{~T}>\mathrm{G}$ splice site variant. $\mathbf{d}$ Sanger sequencing of DNA from healthy control, parents and affected individual III-1, showing heterozygosity and homozygosity of the parents and child (correspondingly) to the identified variant. e Amino acids encoded by the skipped exon 15 are highly conserved throughout evolution

both in movement and at rest and he could not reach for objects. There were decreased movements in the lower limbs. No dysmorphic features were noted. Tongue fasciculations were evident. Muscle tone was low and deep tendon reflexes were decreased. Muscle mass seemed adequate. Further review of systems and physical examinations did not reveal other abnormalities. Brain MRI at the age of 1 year demonstrated cerebellar hypoplasia or atrophy (Fig. 2b). Over the ensuing two years further neurological regression was noted, with inability to purposely move his limbs hands and a decrease in eliciting sounds with minimal capability of communication. He had not achieved milestones such as sitting or standing or talking. Repeated aspirations due to difficulty in eating solid food and slow weight gain led to gastrostomy insertion at two years of age. A progressive decline in the head circumference percentile 


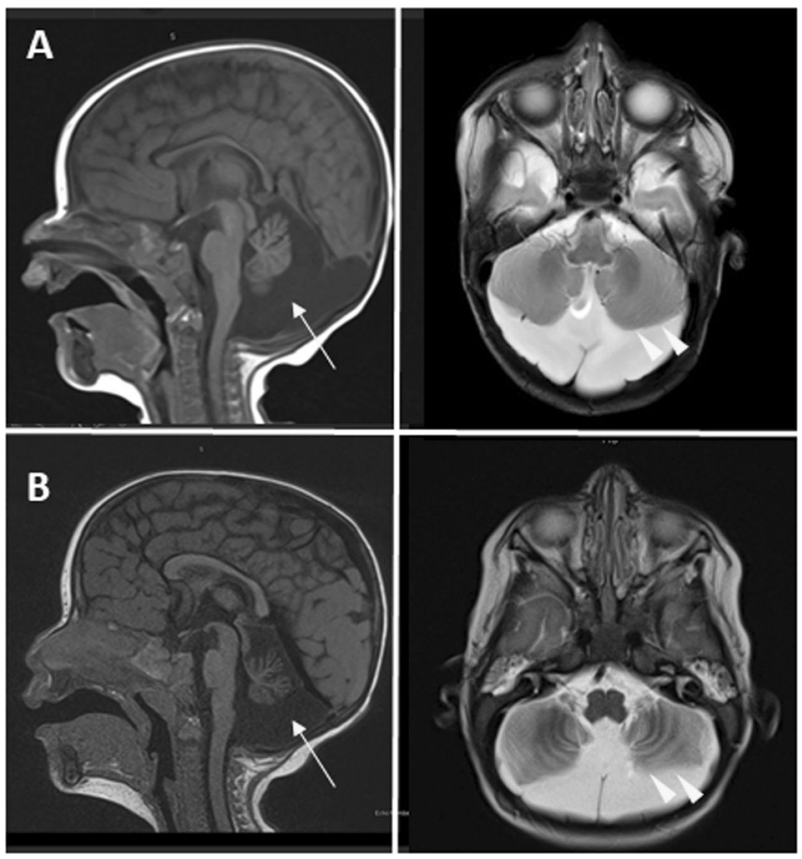

Fig. 2 Brain magnetic resonance imaging (MRI) of affected individuals. Sagittal T1 and axial T2 views demonstrating enlarged CSF spaces, especially in the posterior fossa, with relatively small cerebellar hemispheres and vermis (yellow arrows and arrowheads). a Family 1, individual III-1 at 5 months of age. b Family 2, individual II-6 at 12 months of age

for age was noted (Fig. S1), and at last examination at 45 months of age was measured to be $46 \mathrm{~cm}(-2.7 \mathrm{SD})$. Eyesight and hearing were normal.

\section{Whole exome analysis identified biallelic variants in AGTPBP1}

Whole exome analysis did not reveal any rare variant in a known gene associated with cerebellar hypoplasia or motor neuropathy. However, patient 1 was homozygous for a splice site intronic variant in the $A G T P B P 1$ gene chr9: g.88234128A $>$ C [hg 19]; NM_001286715.1: c.2342+2T $>$ G; r.2256_2342del. This variant was not found in the GnomAD database nor in the in-house database of $\sim 3500$ exomes (Table S2). Parents were both heterozygotes for the variant, and no siblings were available for segregation (Fig. 1a). cDNA generated from the patient blood cells was abnormally short due to skipping of exon 15 (Fig. 1b-d). Exon 15 consists of $87 \mathrm{bp}$ and its loss is predicted to result in the in-frame absence of 29 highly conserved amino acids (Fig. 1e). In addition to the above variant, a homozygous variant in NCAPD3 (c.1031A >T; p.Glu344Val) was identified (Table S2). Variants in NCAPD3, which encodes a condensin complex subunit, have been associated with autosomal recessive primary microcephaly [MIM 617984]. We cannot rule out a potential contribution of this variant to the phenotype although $A G T P B P 1$ seems to be the main contributor.
In patient 2, exome sequencing identified two variants in the AGTPBP1 gene (Fig. 3a, b): c.[2351A>G];[2998C >T]. The first was a maternally inherited variant (chr9: g.88234038T >C [hg19]; NM_001286715.1; c.2351A >G, p. Tyr784Cys), affecting a highly conserved residue (Fig. 3c), and the second was a de novo stopgain variant predicted to cause a premature termination codon (chr9: g.88203274G >A [hg19]; NM_001286715.1; c.2998C >T, p. Arg1000Ter). Paternity was confirmed by short tandem repeats (STR) analysis. Neither variant was found in the GnomAD database nor in the in-house database (Table S2). Segregation studies revealed the c.2351A $>$ G allele in heterozygous state in the mother and three sisters of the proband (Fig. 1a), while no siblings shared the de novo c.2998C $>$ T variant (Fig. 3a).

To determine whether the de novo allele was located in trans to the inherited maternal allele, a fragment encompassing both variants was amplified from cDNA and cloned to separate the alleles. Clones were sequenced by Sanger, and either showed (a) wild-type allele at position r.2351a $>$ g and variant allele at position r.2998c $>$ u, or (b) vice versa, variant allele at position $\mathrm{r} .2351 \mathrm{a}>\mathrm{g}$ and wild-type allele at position r.2998c >u (Fig. 3d). This confirmed that the variants were on two separate alleles, i.e., in trans position. Notably, the r.2998c $>u$ variant allele leading to a premature termination codon was seen in only 1 of 15 clones sequenced, indicating significant nonsense mediated decay of the relevant transcript.

\section{Discussion}

In this study, we report two unrelated individuals with congenital muscle weakness progressing to flaccid tetraparesis, muscle wasting and areflexia. Electrophysiological studies performed in the affected individual in Family 1 were indicative of anterior horn cell disease, and brain neuroimaging showed decreased cerebellar volume. The combination of motor neuropathy and cerebellar hypoplasia/atrophy is suggestive of pontocerebellar hypoplasia type 1 (PCH1). As mentioned above, $\mathrm{PCH} 1$ demonstrates locus heterogeneity, and has been associated with variants in genes encoding several exosome components, genes involved in amyloid- $\beta$ precursor protein (APP) maintenance and a mitochondrial dynamics protein.

Exome sequencing in both patients revealed biallelic variants in $A G T P B P 1$. The first patient had a splice-site variant leading to exon skipping, while the second patient had an inherited variant and a second de novo variant, a rare mechanism for autosomal recessive disease. The encoded protein, ATP/GTP-Binding Protein 1 (OMIM *606830), also known as NNA1 (Nervous system Nuclear protein induced by Axotomy) and CCP1 (Cytosolic 
A

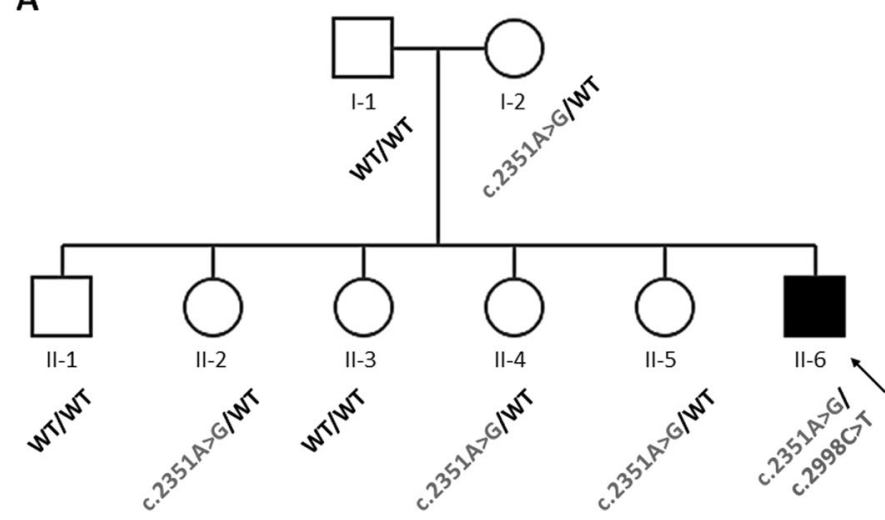

C

$\begin{array}{llll}\text { H.sapiens } & 770 & \text { SGNLRKIQIRKNEY PLILNSDINSNHYHQW } & 800 \\ \text { P.troglodytes } & 678 & \text { SGNLRKVIQIRKNEY PLILNSDINSNHYHQW } & 727 \\ \text { M.mulatta } & 678 & \text { SGNLRKVIQIRKNEY YLILNSDINSNHYHQW } & 727 \\ \text { C.lupus } & 718 & \text { SGNLRKVIQIRKNEY Y DLILNSDINSNHYHQW } & 767 \\ \text { B.taurus } & 718 & \text { SGNLRKVIQIRKNEY YLILNSDINSNHYHQW } & 767 \\ \text { M.musculus } & 710 & \text { SGNLRKVIQIRKSEY YLILNSDINSNHYHQW } & 759 \\ \text { R.norvegicus } & 711 & \text { SGNLRKVIQIRKSEYPLILNSDINSNHYHQW } & 760 \\ \text { G.gallus } & 717 & \text { SGNLRKVIQIRKNEY PLILNSDINSNHYHQW } & 766 \\ \text { D.rerio } & 644 & \text { SGNLRKAIQVRKFEY PLILNSDINSNHYHQW } & 693 \\ \text { X.tropicalis } & 713 & \text { SGNLRKVIQIRKNEYDLILNSDINSNHYHQW } & 762\end{array}$

Fig. 3 Pedigree and molecular analysis of Family 2. a Pedigree indicating the affected child (c.[2351A $>$ G]; [2998C $>$ T] and genotypes of family members. The c. $2351 \mathrm{~A}>\mathrm{G}$ variant is maternally inherited, while c. $2998 \mathrm{C}>\mathrm{T}$ is de novo. b Sanger sequencing of DNA from parents and affected individual 2. c Tyr784 and neighboring amino acids are highly conserved throughout evolution. d Allele separation

Carboxypeptidase 1), catalyzes deglutamylation of polyglutamylated proteins [18]. The protein consists of an ATP/ GTP-binding motif of the P-loop type, a leucine zipper, a nuclear localization signal, a zinc carboxypeptidase signature, and a nucleotide-binding site. It contains multiple canonical phosphorylation sites for protein kinase $\mathrm{C}$, casein kinase II, and cGMP/cAMP-dependent kinases. In addition, it harbors four consensus phosphorylation sites for tyrosine kinases [19].

Polyglutamylation is a post-translational modification in which glutamate side chains of variable lengths are formed on the modified protein. It is evolutionarily conserved from protists to mammals and its most prominent substrate is tubulin $[20,21]$. Polyglutamylated microtubules which accumulate during brain development are involved in synapse vesicle transport or neurite outgrowth through interactions with motor proteins or microtubule-associated proteins, respectively. AGTPBP1 specifically catalyzes the removal of the penultimate glutamate residue from detyrosinated $\alpha$-tubulin, thus generating $\Delta 2$-tubulin. In addition, it shortens posttranslationally generated glutamate side
B
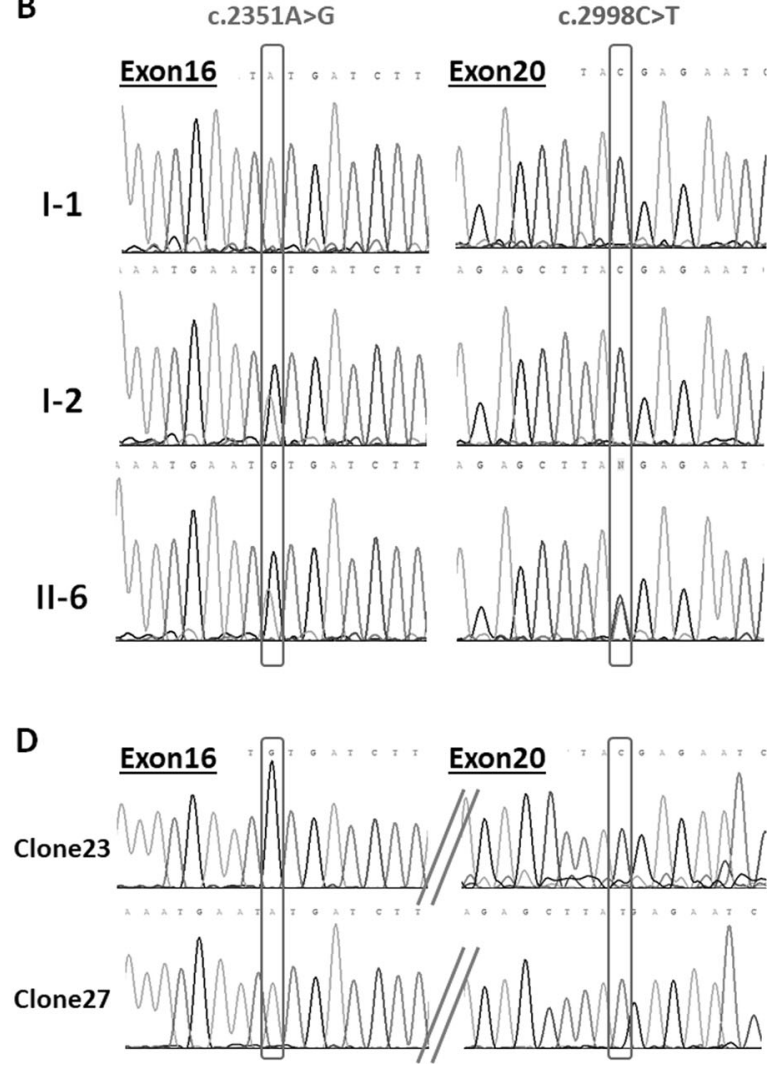

by cloning of a single amplicon of exons 15-21 from cDNA, followed by Sanger sequencing, indicated that the inherited and de novo variants were in trans position. The upper panel (clone 23) shows the variant allele in exon 16 and the wild-type allele in exon 20, while the lower panel (clone 27) shows the wild-type allele in exon 16 and the variant allele in exon 20

chains on tubulin, serving as a tubulin deglutamylase. AGTPBP1 also removes glutamic acids from the $\mathrm{C}$ termini of additional substrates, including myosin light chain kinase 1 (MLCK1) [18].

Variants in $A G T P B P 1$ were previously reported in the mouse Purkinje cell degeneration $(p c d)$ phenotype [21, 22]. Tubulin polyglutamylation was highly increased specifically in the degenerated brain areas, suggesting that microtubule hyperglutamylation is directly linked to neurodegeneration. Downregulating polyglutamylation partially prevented neurodegeneration [23].

Additional spontaneously occurring AGTPBPI mutants are the New Zealand Romney lambs. These animals were reported 20 years ago because of their lower motor neuronlike disease manifesting by progressive weakness and tetraparesis which started after the first week of life [24]. Predominant histological lesions were degeneration and loss of neurons in the ventral horns of the spinal cord and the brain stem, Wallerian degeneration of the motor nerves and denervation atrophy of skeletal muscles fibers. Of note, cerebellar histology was intact at 4 weeks of age. 
Subsequent molecular analysis disclosed a homozygous variant in the sheep ortholog $A G T P B P 1$, affecting a highly conserved residue in the catalytic domain of the encoded protein [25]. A homozygous variant (p.Arg970Trp) was previously reported in a human child with global developmental delay and brain atrophy [26], yet no further details were available.

Thus our data suggest that biallelic variants in AGTPBPI are associated with $\mathrm{PCH}$ and SMA-like disease; this phenotype is reminiscent of the Purkinje cell degeneration $(p c d)$ mouse and the motor-neuron disease in the New Zealand Romney sheep [21, 24]. Most of our patients' symptoms likely derived from Purkinje cell degeneration and motor neuronopathy. We propose that these degenerative processes result from impaired deglutamylation of central nervous system (CNS) specific tubulin. Interestingly, $A G T P B P 1$ was identified as the most significant gene coexpressed with C9orf72, a gene implicated in familial and sporadic amyotrophic lateral sclerosis and frontotemporal dementia, and may serve as a C9orf72 interacting partner in regulation of neuronal function within the CNS [27]. However, additional studies are required in order to elucidate the underlying pathogenic mechanism of AGTPBPIrelated disease.

The treatment of affected individuals with AGTPBPIrelated disease is at present symptomatic and supportive. Presence of robust animal models for this complex disorder may assist research for therapeutic options such as downregulating polyglutamylation in affected individuals.

\section{Accession numbers}

The ClinVar accession numbers for the DNA variant data reported in this manuscript are SCV000844952, SCV000844953, and SCV000864167.

Acknowledgements The authors wish to thank the families for their participation in this study.

\section{Compliance with ethical standards}

Conflict of interest The authors declare that they have no conflict of interest.

Publisher's note: Springer Nature remains neutral with regard to jurisdictional claims in published maps and institutional affiliations.

\section{References}

1. van Dijk T, Baas F, Barth PG, Poll-The BT. What's new in pontocerebellar hypoplasia? An update on genes and subtypes. Orphanet J Rare Dis. 2018;13:92.

2. Norman RM. Cerebellar hypoplasia in Werdnig-Hoffmann disease. Arch Dis Child. 1961;36:96-101.
3. Goutières F, Aicardi J, Farkas E. Anterior horn cell disease associated with pontocerebellar hypoplasia in infants. J Neurol Neurosurg Psychiatry. 1977;40:370-8.

4. Kamoshita S, Takei Y, Miyao M, Yanagisawa M, Kobayashi S, Saito K. Pontocerebellar hypoplasia associated with infantile motor neuron disease (Norman's disease). Pediatr Pathol. 1990;10:133-42.

5. Wan J, Yourshaw M, Mamsa H, Rudnik-Schöneborn S, Menezes MP, Hong JE, et al. Mutations in the RNA exosome component gene EXOSC 3 cause pontocerebellar hypoplasia and spinal motor neuron degeneration. Nat Genet. 2012;44:704-8.

6. Rudnik-Schöneborn S, Senderek J, Jen JC, Houge G, Seeman P, Puchmajerová A, et al. Pontocerebellar hypoplasia type 1: clinical spectrum and relevance of EXOSC3 mutations. Neurology. 2013;80:438-46.

7. Eggens VR, Barth PG, Niermeijer JM, Berg JN, Darin N, Dixit A, et al. EXOSC3 mutations in pontocerebellar hypoplasia type 1: novel mutations and genotype-phenotype correlations. Orphanet $\mathrm{J}$ Rare Dis. 2014;9:23.

8. Boczonadi V, Müller JS, Pyle A, Munkley J, Dor T, Quartararo J. et al. EXOSC8 mutations alter mRNA metabolism and cause hypomyelination with spinal muscular atrophy and cerebellar hypoplasia. Nat Commun. 2014;5:4287

9. Burns DT, Donkervoort S, Müller JS, Knierim E, BharuchaGoebel D, Faqeih EA, et al. Variants in EXOSC9 disrupt the RNA exosome and result in cerebellar atrophy with spinal motor neuronopathy. Am J Hum Genet. 2018;102:858-73.

10. Renbaum P, Kellerman E, Jaron R, Geiger D, Segel R, Lee M, et al. Spinal muscular atrophy with pontocerebellar hypoplasia is caused by a mutation in the VRK1 gene. Am J Hum Genet. 2009;85:281-9.

11. Gonzaga-Jauregui C, Lotze T, Jamal L, Penney S, Campbell IM, Pehlivan D, et al. Mutations in VRK1 associated with complex motor and sensory axonal neuropathy plus microcephaly. JAMA Neurol. 2013;70:1491-8.

12. Stoll M, Teoh H, Lee J, Reddel S, Zhu Y, Buckley M, et al. Novel motor phenotypes in patients with VRK1 mutations without pontocerebellar hypoplasia. Neurology. 2016;87:65-70.

13. Wan J, Steffen J, Yourshaw M, Mamsa H, Andersen E, RudnikSchöneborn S, et al. Loss of function of SLC25A46 causes lethal congenital pontocerebellar hypoplasia. Brain. 2016;139:2877-90.

14. Abrams AJ, Hufnagel RB, Rebelo A, Zanna C, Patel N, Gonzalez MA, et al. Mutations in SLC25A46, encoding a UGO1-like protein, cause an optic atrophy spectrum disorder. Nat Genet. 2015;47:926-32.

15. Vinograd-Byk H, Sapir T, Cantarero L, Lazo PA, Zeligson S, Lev $\mathrm{D}$, et al. The spinal muscular atrophy with pontocerebellar hypoplasia gene VRK1 regulates neuronal migration through an amyloid- $\beta$ precursor protein-dependent mechanism. J Neurosci. 2015;35:936-42.

16. Janer A, Prudent J, Paupe V, Fahiminiya S, Majewsky J, Sgarioto $\mathrm{N}$, et al. $S L C 25 A 46$ is required for mitochondrial lipid homeostasis and cristae maintenance and is responsible for Leigh syndrome. EMBO Mol Med. 2016;8:1019-38.

17. Ta-Shma A, Zhang K, Salimova E, Zernecke A, Sieiro-Mosti D, Stegner D, et al. Congenital valvular defects associated with deleterious mutations in the PLD1 gene. J Med Genet. 2017;54:278-86.

18. Rogowski K, van Dijk J, Magiera MM, Bosc C, Deloulme JC, Bosson A, et al. A family of protein-deglutamylating enzymes associated with neurodegeneration. Cell. 2010;143:564-78.

19. Harris A, Morgan JI, Pecot M, Soumare A, Osborne A, Soares HD. Regenerating motor neurons express Nna1, a novel ATP/ GTP-binding protein related to zinc carboxypeptidases. Mol Cell Neurosci. 2000;16:578-96. 
20. Fukushima N, Furuta D, Hidaka Y, Moriyama R, Tsujiuchi T. Post-translational modifications of tubulin in the nervous system. $\mathrm{J}$ Neurochem. 2009;109:683-93.

21. Fernandez-Gonzalez A, La Spada AR, Treadaway J, Higdon JC, Harris BS, Sidman RL, et al. Purkinje cell degeneration (pcd) phenotypes caused by mutations in the axotomy-induced gene, Nna1. Science. 2002;295:1904-6.

22. Wu HY, Wang T, Li L, Correia K, Morgan JI. A structural and functional analysis of Nnal in Purkinje cell degeneration (pcd) mice. FASEB J. 2012;26:4468-80.

23. Wu HY, Rong Y, Correia K, Min J, Morgan JI. Comparison of the enzymatic and functional properties of three cytosolic carboxypeptidase family members. J Biol Chem. 2015;290:1222-32.
24. Anderson PD, Parton KH, Collett MG, Sargison ND, Jolly RD. A lower motor neuron disease in newborn Romney lambs. $\mathrm{N} Z \mathrm{~V}$ Vet J. 1999;47:112-4.

25. Zhao X, Onteru SK, Dittmer KE, Parton K, Blair HT, Rothschild $\mathrm{MF}$, et al. A missense mutation in $A G T P B P 1$ was identified in sheep with a lower motor neuron disease. Heredity. 2012;109:156-62.

26. Monies D, Abouelhoda M, AlSayed M, Alhassnan Z, Alotaibi M, Kayyali $\mathrm{H}$, et al. The landscape of genetic diseases in Saudi Arabia based on the first 1000 diagnostic panels and exomes. Hum Genet. 2017;136:921-39.

27. Kitano S, Kino Y, Yamamoto Y, Takitani M, Miyoshi J, Ishida T, et al. Bioinformatics data mining approach suggests coexpression of $A G T P B P 1$ with an ALS-linked gene C9orf72. J Cent Nerv Syst Dis. 2015;7:15-26. 